\title{
Consorcio Mexicano de Centros de Estudios del APEC
}

DOI: 10.32870/mycp.v7i21.221

$\mathrm{E}$ 1 Mecanismo de Cooperación Económica de Asia Pacífico (APEC) fue establecido en 1989 como un mecanismo intergubernamental para promover el desarrollo económico de la región de Asia Pacífico mediante el comercio y la inversión. Actualmente, 21 economías de la región pertenecen al APEC.

El APEC promueve un regionalismo abierto, es decir, la región no introduce medidas vinculadas al comercio y la inversión para discriminar economías que no pertenezcan al APEC. Este regionalismo se basa en un proceso de toma de decisiones por consenso, voluntario, comprehensivo, flexible, transparente y con esquemas para economías en desarrollo y desarrolladas.

En 1994, en Bogor, Indonesia, se determinaron las metas de largo plazo del APEC: establecer una región libre y abierta de comercio e inversión en 2010 para las economías desarrolladas y en 2020 para las economías en desarrollo. Dos son los pilares del APEC:

a) La facilitación y liberalización del comercio e inversión (TILF, por sus siglas en inglés) mediante acuerdos unilaterales concertados. Los compromisos de los países hacia la liberalización están contenidos en los planes de acción individual (IAP) y en los planes de acción colectiva (CAP).

b) La cooperación económica y técnica (ЕСОTECH, por sus siglas en inglés), la cual implica una mayor cooperación para sostener el crecimiento y desarrollo de la región. Esta cooperación se basa en el reconocimiento de la diversidad de la región y en el compromiso de diálogo abierto y consenso con igual respeto para todos los

* Profesora investigadora del Departamento de Estudios del Pacífico, de la Universidad de Guadalajara.

ORCID http://orcid.org/0000-0003-4926-0594
Melba E. Falck Reyes*

participantes sobre la base de intercambios de consulta no formales.

Una de las fortalezas del APEC es la rotación anual de su sede. Cada año, una economía asume la presidencia del APEC y tiene un papel crucial al influir en la dirección del desarrollo del mecanismo, además de la posición privilegiada de inyectar nuevas ideas y de ampliar la agenda o profundizar ciertos aspectos.

México es miembro del APEC desde 1993, y en 2002 México fue sede del mecanismo. La temática propuesta por México en ese año se centró en la expansión de los beneficios del crecimiento y el desarrollo económico, a través de:

1) La construcción de capacidades, haciendo hincapié en la promoción de las pequeñas y medianas empresas, así como las microempresas.

2) Hacer del APEC un foro más relevante para la comunidad mediante el fomento de la participación de mujeres y jóvenes.

\section{Consorcio de centros de estudios del APEC}

Los centros de estudios del APEC (CEA) tienen como objetivo dar seguimiento al desarrollo del mecanismo de una manera sistemática, y aumentar las capacidades analíticas independientes para la evaluación del proceso. En la actualidad, 19 de las 21 economías que conforman el APEC poseen centros de estudios. El consorcio internacional está constituido por alrededor de 100 centros de estudios que tienen su sede en distintas universidades y centros de investigación. En México actualmente hay cuatro centros de estudios del APEC. 


\section{El Consorcio Mexicano de Centros de Estudios del APEC (CONMEX-CEA)}

Este consorcio fue establecido en enero de 2003 y está constituido por los centros de estudio del APEC reconocidos oficialmente por el gobierno mexicano y registrados en el secretariado del APEc. La Secretaría Técnica del Consorcio tiene actualmente como sede el Departamento de Estudios del Pacífico (DEP) y el Centro de Estudios del APEC de la Universidad de Guadalajara.

\section{Actualmente hay cuatro CEA en México:}

El Centro de Estudios del APEC en la Universidad de Baja California Sur (UBCS).

El Programa de Estudios del APEC de El Colegio de México (colmex).

El Centro Universitario de Estudios de la Cuenca del Pacífico, de la Universidad de Colima (CUECP).

El Departamento de Estudios del Pacífico (DEP), del Centro Universitario de Ciencias Sociales y Humanidades (CUCSH), de la Universidad de Guadalajara (UdeG).

Los cuatro CEA formalizaron su relación con la firma de un convenio de colaboración el 27 de marzo de 2004.

\section{La misión del consorcio}

- Promover la cooperación de la educación superior y la investigación avanzada, así como facilitar el intercambio cultural e intelectual en la región Asia Pacífico, reconociendo la importancia de los vínculos educacionales en el desarrollo de un sentido de comunidad y fomentando el entendimiento en la diversidad de la región.

- Apoyar el proceso del APEC llevando a cabo investigación avanzada, interdisciplinaria, colaboradora y relevante a las políticas públicas, en aquellas temáticas importantes para el APEC y desde una perspectiva independiente y de largo plazo.

- Promover el intercambio estudiantil, académico y de investigación entre las economías del APEC, e incentivar la capacitación y otros programas educativos dirigidos a las necesidades de la región.

- Gestionar la participación informada de otros sectores de la sociedad, especialmente los jóvenes, el sector privado-empresarial, las organizaciones no gubernamentales (ONG) y los medios de comunicación, tanto en diálogos como en estudios relacionados con el APEC.

El trabajo académico del consorcio cubre las cuatro áreas sustantivas del quehacer académico: investigación, docencia, difusión y extensión, así como la cooperación interinstitucional aprovechando las fortalezas de cada centro de estudios y respetando la autonomía en las líneas de investigación.

El Consorcio Mexicano está conformado por 24 investigadores, $50 \%$ con doctorado y el resto con maestría. Actualmente, en el consorcio se trabajan siete líneas de investigación que incluyen 26 proyectos vinculados a la Cuenca del Pacífico y que se centran en procesos de integración (3), agricultura (3), liberalización financiera (2), pequeñas y medianas empresas (3) y propiedad intelectual (3), entre otros.

Los centros del APEC que conforman el consorcio tienen los siguientes programas de posgrado relacionados con la Cuenca del Pacífico:

1. Doctorado en Relaciones Internacionales Transpacíficas, Universidad de Colima.

2. Doctorado y Maestría en Estudios de Asia, Colmex.

3. Maestría en Ciencias Sociales con especialidad en Relaciones Internacionales y Estudios del Pacífico, Universidad de Guadalajara.

Los miembros del consorcio publican actualmente un anuario, dos revistas y una se- 
rie de documentos de trabajo, con el fin de difundir el conocimiento de la región no sólo entre la comunidad académica sino entre el público en general:

- Anuario Asia Pacífico, Centro de Estudios de Asia y África de El colmex.

- México y la Cuenca del Pacífico (Dep, Universidad de Guadalajara).

- Aportes: revista mexicana de estudios sobre la Cuenca del Pacífico, Centro Universitario de Estudios de la Cuenca del Pacífico, Universidad de Colima.

- Working Papers, APEC Studies Program, EL COLMEX.

Adicionalmente, el consorcio tiene como uno de sus objetivos contribuir a la creación de centros de estudios del APEC en las instituciones de educación superior (IES) públicas y privadas del país, y la vinculación con los sectores gubernamental, social y empresarial.

La máxima autoridad del consorcio es su Consejo, integrado por los rectores o, en su representación, los responsables de los CEA; dicho consejo se reunirá una vez al año. Los responsables de los CEA miembros del consorcio se reunirán dos veces al año. Para fines operacionales, se estableció una Secretaría Técnica que tendrá como sede uno de los CEA y que será rotativa por un período de dos años. mi: 